\title{
Multi-scale modelling of coastal, shelf and global ocean dynamics
}

\author{
Eric Deleersnijder • Vincent Legat • Pierre F. J. Lermusiaux
}

Received: 12 November 2010 / Accepted: 12 November 2010/Published online: 27 November 2010

(C) Springer-Verlag 2010

\section{Introduction}

Methods for widening the range of resolved scales (i.e. performing multi-scale simulations) in ocean sciences and engineering are developing rapidly, now allowing multiscale ocean dynamics studies. Having recourse to grid nesting has been and still is a popular method for increasing marine models' resolution when and where needed and for easily allowing the use of different dynamics at different resolution. However, this is not the only way to achieve this goal. Various techniques for modifying locally the grid resolution or dealing with complex-geometry domains are

Responsible Editor: Jörg-Olaf Wolff

E. Deleersnijder $\cdot$ V. Legat

Institute of Mechanics, Materials and Civil Engineering (iMMC),

Université catholique de Louvain (UCL),

4 Avenue G. Lemaître,

1348 Louvain-la-Neuve, Belgium

E. Deleersnijder $(\square)$

Earth and Life Institute (ELI), G. Lemaître Centre for Earth and

Climate Research (TECLIM),

Université catholique de Louvain (UCL),

2 Chemin du Cyclotron,

1348 Louvain-la-Neuve, Belgium

e-mail: eric.deleersnijder@uclouvain.be

V. Legat

G. Lemaitre Centre for Earth and Climate Research (TECLIM),

Université catholique de Louvain,

2 Chemin du Cyclotron,

1348 Louvain-la-Neuve, Belgium

\section{P. F. J. Lermusiaux}

Department of Mechanical Engineering,

Massachusetts Institute of Technology,

Room 5-207B, 77 Massachusetts Avenue,

Cambridge, MA 02139-4307, USA available. For instance, composite, structured grids and unstructured meshes offer an almost infinite geometrical flexibility.

This special issue focuses on multi-scale modelling of coastal, shelf and global ocean dynamics, including the development of new methodologies and schemes and their applications to ocean process studies. Several articles focus on numerical aspects of unstructured mesh space discretisation. Danilov (2010) shows that the noise developing on triangular meshes on which the location of the variables is inspired by Arakawa's C-grid is the largest for regimes close to geostrophic balance. The noise can be reduced by specific operators but cannot be entirely suppressed, "making the triangular C-grid a suboptimal choice for large-scale ocean modelling". Then, the companion articles of Blaise et al. (2010) and Comblen et al. (2010) describe the space and time discretisation of a three-dimensional, baroclinic, finite element model based on the discontinuous Galerkin (DG) technique. This is a significant step forward in the field of finite element ocean modelling, though this model cannot yet be regarded as suitable for tackling realistic applications. Ueckermann and Lermusiaux (2010) also consider DG finite element techniques, focusing on biological-physical dynamics in regions with complex bathymetric features. They compare low- to high-order discretisations, both in time and space, for regimes in which biology dominates, advection dominates or terms are balanced. They find that higher-order schemes on relatively coarse grids generally perform better than low-order schemes on fine grids. Kleptsova et al. (2010) assess various advection schemes for $z$-coordinate, threedimensional models in which flooding and drying is taken into account. In this study, the ability to conserve momentum is regarded as the main criterion for selecting a suitable method. On the other hand, Maßmann (2010) 
assesses automatic differentiation for obtaining the adjoint of an unstructured mesh, tidal model of the European continental shelf.

Two articles deal with grid nesting. Nash and Hartnett (2010) introduce a flooding and drying method that can be used in structured, nested grid systems. This can be regarded as an alternative to flooding and drying techniques that are being developed for unstructured mesh models (e.g. Kärnä et al. 2010). Then, Haley and Lermusiaux (2010) derive conservative time-dependent structured finite volume discretisations and implicit two-way embedded schemes for primitive equations with the intent to resolve tidal-to-mesoscale processes over large multi-resolution telescoping domains with complex geometries including shallow seas with strong tides, steep shelf breaks and deep ocean interactions. The authors present realistic simulations with data assimilation in three regions with diverse dynamics and show that their developments enhance the predictive capability, leading to better match with ocean data.

Various multi-scale, realistic simulations are presented. Using a finite element ice model and a slab ocean as in Lietaer et al. (2008), Terwisscha van Scheltinga et al. (2010) model the Canadian Arctic Archipelago, focusing on the pathways for freshwater and sea-ice transport from the Arctic Ocean to the Labrador Sea and the Atlantic Ocean. The unstructured mesh can represent the complex geometry and narrow straits at high resolution and allows improving transports of water masses and sea ice. Walters et al. (2010) have recourse to an unstructured mesh model to study tides and current in Greater Cook Strait (New Zealand). They identify the mechanisms causing residual currents. By means of the unstructured mesh Finite Volume Coastal Ocean Model (FVCOM), Wang et al. (2010) study the hydrodynamics of the Bohai Sea. Xu et al. (2010) simulate coastal and urban inundation due to storm surges along US East and Gulf Coasts. A sensitivity analysis reveals the importance of precise topographic data and the need for a bottom drag coefficient accounting for the presence of mangroves. Finally, Yang and Khangaonkar (2010) resort to FVCOM to simulate the three-dimensional circulation of Puget Sound, a large complex estuary system in the Pacific Northwest coastal ocean, including variable forcing from tides, the atmosphere and river inflows. Comparisons of model estimates with measurements for tidal elevation, velocity, temperature and salinity are deemed to be promising, from larger-scale circulation features to nearshore tide flats.

This special issue suggests that numerical techniques for multi-scale space discretisation are progressively becoming mature. One direction for future progress lies in the improvement of time discretisation methods for the new generation models, so that they can successfully compete with finite difference, structured mesh models based on (almost) constant resolution grids that have been developed and used over the past 40 years (e.g. Griffies et al. 2009).

Acknowledgements The guest editors are indebted to the authors and anonymous reviewers for their contributions to this special issue. Eric Deleersnijder is a research associate with the Belgian Fund for Scientific Research (F.R.S.-FNRS). The contributions of Eric Deleersnijder and Vincent Legat to this special issue were achieved in the framework of the project "Taking up the challenges of multi-scale marine modelling", which is funded by the Communauté Française de Belgique under contract ARC 10/15-028 and the Interuniversity Attraction Pole Timothy (www.climate.be/timothy). The latter is funded by BELSPO (www. belspo.be) under contract IAP6.13. Pierre Lermusiaux is very grateful to the Office of Naval Research for support under grants N00014-07-1-1061 and N00014-08-1-1097 to the Massachusetts Institute of Technology. Some of the articles of this special issue derive from presentations made at the 8th International Workshop on Unstructured Mesh Numerical Modelling of Coastal, Shelf and Ocean Flows (16-18 September 2009, Louvain-la-Neuve, Belgium, www.uclouvain.be/umm2009), for which the F.R.S.-FNRS and the contract ARC 04/09-316 (Communauté Française de Belgique) provided financial support.

\section{References}

Blaise S, Comblen R, Legat V, Remacle J-F, Deleersnijder E, Lambrechts J (2010) A discontinuous finite element baroclinic marine model on unstructured prismatic meshes. Part I: space discretization. Ocean Dynamics (this issue)

Comblen R, Blaise S, Legat V, Remacle J-F, Deleersnijder E, Lambrechts J (2010) A discontinuous finite element baroclinic marine model on unstructured prismatic meshes. Part II: implicit/ explicit time discretization. Ocean Dynamics (this issue)

Danilov S (2010) On utility of triangular C-grid type discretization for numerical modeling of large-scale ocean. Ocean Dynamics (this issue)

Griffies SM, Adcroft AJ, Banks H, Böning CW, Chassignet EP, Danabasoglu G, Danilov S, Deleersnijder E, Drange H, England MH, Fox-Kemper B, Gerdes R, Gnanadesikan A, Greatbatch RJ, Hallberg RW, Hanert E, Harrison MJ, Legg SA, Little CM, Madec G, Marsland S, Nikurashin M, Pirani A, Simmons HL, Schröter J, Samuels BL, Treguier AM, Toggweiler JR, Tsujino H, Vallis GK, White L (2009) Problems and prospects in large-scale ocean circulation models. In: Hall J, Harrison DE, Stammer D (eds) Proceedings of the OceanObs'09 conference: sustained ocean observations and information for society. ESA Publication WPP-306. ESA, Venice, pp 21-25

Haley PJ Jr, Lermusiaux PFJ (2010) Multiscale two-way embedding schemes for free-surface primitive-equations in the "Multidisciplinary simulation, estimation and assimilation system". Ocean Dynamics (this issue)

Kärnä T, de Brye B, Gourgue O, Lambrechts J, Comblen R, Legat V, Deleersnijder E (2010) A fully implicit wetting-drying method for DG-FEM shallow water models, with an application to the Scheldt Estuary. Computer Methods in Applied Mechanics and Engineering. doi:10.1016/j.cma.2010.07.001

Kleptsova O, Stelling GS, Pietrzak JD (2010) An accurate momentum advection scheme for a $z$-level coordinate model. Ocean Dynamics (this issue)

Lietaer O, Fichefet T, Legat V (2008) The effects of resolving the Canadian Arctic Archipelago in a finite element sea ice model. Ocean Model 24:140-152 
Maßmann S (2010) Sensitivities of an adjoint, unstructured mesh, tidal model on the European Continental Shelf. Ocean Dynamics (this issue)

Nash S, Hartnett M (2010) Nested circulation modelling of inter-tidal zones: details of a nesting approach incorporating moving boundaries. Ocean Dynamics (this issue)

Terwisscha van Scheltinga AD, Myers PG, Pietrzak JD (2010) A finite element sea ice model of the Canadian Arctic Archipelago. Ocean Dynamics (this issue)

Ueckermann MP, Lermusiaux PFJ (2010) High order schemes for 2D unsteady biogeochemical ocean models. Ocean Dynamics (this issue)
Walters RA, Gillibrand PA, Bell R.G, Lane EM (2010) A study of tides and currents in Cook Strait, New Zealand. Ocean Dynamics (this issue)

Wang J, Shen Y, Guo Y (2010) Seasonal circulation and influence factors of the Bohai Sea: a numerical study based on Lagrangian particle tracking method. Ocean Dynamics (this issue)

Xu H, Zhang K, Shen J, Li Y (2010) Storm surge simulation along the U.S. East and Gulf Coasts using a multi-scale numerical model approach. Ocean Dynamics (this issue)

Yang Z, Khangaonkar T (2010) Multi-scale modeling of Puget Sound using an unstructured-grid coastal ocean model: from tide flats to estuaries and coastal waters. Ocean Dynamics (this issue) 